\title{
Effects of temporal features and order on the apparent duration of a visual stimulus
}

\author{
Aurelio Bruno $^{1}{ }^{*}$, Inci Ayhan ${ }^{1}$ and Alan Johnston ${ }^{1,2}$ \\ 1 Department of Cognition, Perception and Brain Sciences, University College London, London, UK \\ ${ }^{2}$ Centre for Mathematics and Physics in the Life Sciences and Experimental Biology, University College London, London, UK
}

\author{
Edited by: \\ Lars Muckli, University of Glasgow, \\ UK \\ Reviewed by: \\ Andreas Keil, University of Florida, \\ USA \\ Duje Tadin, University of Rochester, \\ USA \\ *Correspondence: \\ Aurelio Bruno, Department of \\ Cognition, Perception and Brain \\ Sciences, University College London, \\ 26 Bedford Way, London WC1H OAP, \\ UK \\ e-mail: a.bruno@ucl.ac.uk
}

The apparent duration of a visual stimulus has been shown to be influenced by its speed. For low speeds, apparent duration increases linearly with stimulus speed. This effect has been ascribed to the number of changes that occur within a visual interval. Accordingly, a higher number of changes should produce an increase in apparent duration. In order to test this prediction, we asked subjects to compare the relative duration of a $10-\mathrm{Hz}$ drifting comparison stimulus with a standard stimulus that contained a different number of changes in different conditions. The standard could be static, drifting at $10 \mathrm{~Hz}$, or mixed (a combination of variable duration static and drifting intervals). In this last condition the number of changes was intermediate between the static and the continuously drifting stimulus. For all standard durations, the mixed stimulus looked significantly compressed ( $20 \%$ reduction) relative to the drifting stimulus. However, no difference emerged between the static (that contained no changes) and the mixed stimuli (which contained an intermediate number of changes). We also observed that when the standard was displayed first, it appeared compressed relative to when it was displayed second with a magnitude that depended on standard duration. These results are at odds with a model of time perception that simply reflects the number of temporal features within an interval in determining the perceived passing of time.

Keywords: perceived duration, speed, temporal change, psychophysics, stimulus order

\section{INTRODUCTION}

The explicit encoding of the duration of events within the subsecond range is crucial for a number of everyday tasks from timing action such as deciding when to step onto an escalator or when to move off at traffic lights to picking up social signals encoded in the duration of mutual gaze. However, the mechanisms underlying the timing of events under 1 or $2 \mathrm{~s}$ are still obscure. A substantial number of studies have shown that the apparent duration of a visual stimulus in the order of milliseconds can be distorted not only by generic factors, such as stimulus novelty (Pariyadath and Eagleman, 2007, 2008) or attention (Tse et al., 2004; Cicchini and Morrone, 2009), but also by visually specific manipulations, such as adaptation to visual motion (Johnston et al., 2006, 2008; Burr et al., 2007; Ayhan et al., 2009, 2011; Bruno et al., 2010), contrast (Bruno and Johnston, 2010), and reduced illumination (Bruno et al., 2011). These observations have led some authors to question the ability of the classic pacemaker-accumulator model (Creelman, 1962; Treisman, 1963; Treisman et al., 1990) to fully account for the perceptual encoding of temporal intervals. Therefore, alternative models based on coding efficiency (Eagleman and Pariyadath, 2009) or on a predict-and-compare strategy (Johnston, 2010) have recently been proposed.

The relationship between motion, speed, and perceived duration has been extensively investigated. Early studies revealed that moving stimuli tend to be seen as having a longer duration than stationary ones (Brown, 1931; Roelofs and Zeeman, 1951; Goldstone and Lhamon, 1974; Lhamon and Goldstone, 1974).
This observation, obtained with stimuli that were at most a few seconds long, was replicated for stimuli lasting several seconds (Brown, 1995) or even minutes (Leiser et al., 1991). Some of these studies also showed a certain degree of dependency of duration estimation on stimulus speed: the faster the stimulus, the bigger the duration overestimation. In the sub-second range, two recent studies investigated this relationship in detail. Kanai et al. (2006), using a variety of visual stimuli including squares, random dot patterns, expanding gratings, and flickering Gaussian blobs, reported that apparent duration increased with stimulus temporal frequency, rather than speed, but only up to $4-8 \mathrm{~Hz}$. Kaneko and Murakami (2009), using drifting Gabors, observed that perceived duration is positively related to speed, rather than temporal frequency, and the effect does not seem to saturate at high speed levels.

Most of the above-mentioned studies tended to explain the effect of speed (or temporal frequency) on apparent duration by referring to a change model (Fraisse, 1963). According to this view, the number of changes that occur within an interval provides a cue to the passage of time (Block and Reed, 1978; Poynter, 1983, 1989; Poynter and Homa, 1983). An interval containing fast motion or fast temporal change would be represented as having more temporal features than a slower stimulus and therefore would be perceived as having a longer duration. In contrast to this passive view of the effect of content on perceived duration, we can consider an active "predict-and-compare" content-dependant clock in which the content of the interval is intrinsic to the measurement of its duration (Johnston, 2010). 
The aim of the current paper is to test the predictions of the change model, we measured perceived duration for static, drifting, or mixed (where static and drifting intervals were interleaved) stimuli that, according to the change model, should contain a different number of changes and therefore be judged as having different durations. We found that the mixed stimulus was consistently perceived as compressed relative to the drifting one but no different in duration to the static stimulus (no change) across duration ranges $(600,1200$, and $2400 \mathrm{~ms})$, without any substantial changes in the accuracy of the duration judgments.

Presenting a pair of stimuli sequentially can induce perceptual biases that depend on their position in the sequence. The observation that the magnitude of the first test is systematically underestimated dates back to Fechner (Fechner, 1860; Woodrow, 1951; Woodworth and Schlosberg, 1954). Consistent with these early results, compression has been reported in the duration domain for the first relative to the second of a pair of brief intervals (Jamieson and Petrusic, 1975). In the present study, we compared apparent duration and accuracy dependence on presentation order across a more extended range of durations. We observed that the difference between first and second tests increased with duration, while the difference in accuracy remained constant.

\section{MATERIALS AND METHODS \\ APPARATUS}

Stimuli were displayed, in a darkened room, on a $19^{\prime \prime}$ Sony Trinitron Multiscan 500PS, with a refresh rate of $100 \mathrm{~Hz}$, driven by a
VSG2/5 visual stimulus generator (Cambridge Research Systems). Stimuli were viewed from a distance of $57 \mathrm{~cm}$.

\section{STIMULI AND PROCEDURE}

Fifteen subjects participated in the experiment (2 authors and 13 naives). All of them had normal or corrected-to-normal vision. Subjects fixated a spot in the center of the screen and judged the relative duration of two stimuli that were displayed sequentially in the near periphery (see Figure 1B). Stimuli were vertically oriented sinusoidal gratings modulated in luminance (spatial frequency $=1 \mathrm{cycle} /{ }^{\circ}$, diameter: $5^{\circ}$ of visual angle, Michelson contrast: $100 \%$, centered $5^{\circ}$ to the right and to the left of the center of the monitor). The duration of one of the stimuli (the standard) was fixed across trials, while the duration of the other stimulus (the comparison) varied across trials in order to generate a psychometric function. We used three standard durations in different sessions: 600,1200 , and $2400 \mathrm{~ms}$. The duration of the comparison varied in seven steps, ranging from $0.2 \times$ standard duration to $2.67 \times$ standard duration. The comparison stimulus always drifted at $10 \%$ across conditions (its phase was randomized on a trialby-trial basis). It was essential that the comparison stimulus was always the same across stimulus types (static, drifting, mixed) in order to have at least two direct comparisons (static vs. drifting and mixed vs. drifting, in this case) between stimuli with different numbers of temporal changes. We chose to use the drifting grating as comparison, however one of the other stimulus types would have served as well, since our analysis was focused on relative duration across the three conditions. The drifting vs. drifting condition

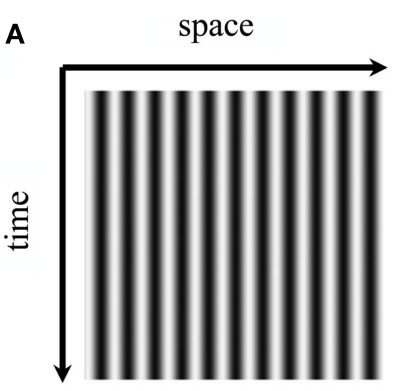

Static

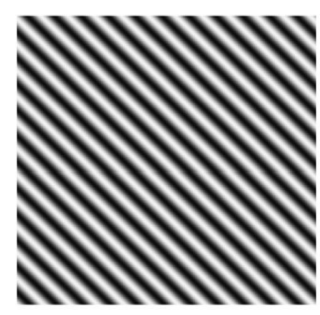

Drifting

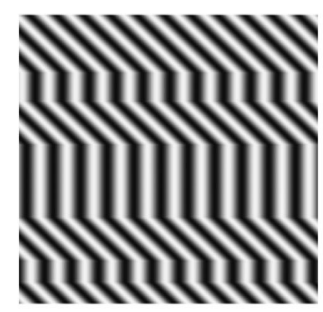

Mixed
B

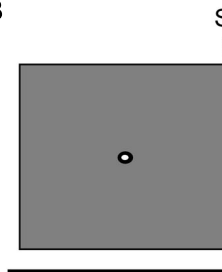

Static, Drifting or Mixed 600,1200 or $2400 \mathrm{~ms}$

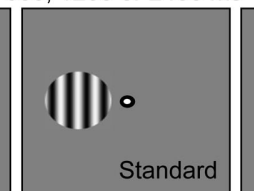

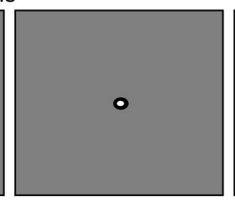

Drifting

Variable duration

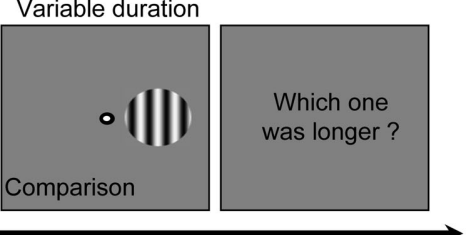

time

FIGURE 1 | Schematic representations of the stimuli and the task. (A) Space-time representation of the three standard stimulus types used in the experiment. The standard intervals contained luminance-modulated sinusoidal gratings that could be, in different sessions, static, drifting at $10 \mathrm{~Hz}$, or composed by an alternation of static and drifting subintervals whose duration varied from interval to interval (see Materials and Methods for a more detailed description). (B)
Subjects had to keep fixation on the middle of the screen while two stimuli (the standard, fixed duration, and the comparison, with variable duration across trials) were sequentially displayed on either side of the fixation spot. Presentation order and spatial positions of standard and comparison were randomized on a trial-by-trial basis. At the end of each trial, subjects had to report which stimulus appeared to stay on for the longer duration by pressing a button. 
provided us with a baseline condition, in which we compared stimuli with the same number of temporal changes. In different sessions the standard could be static, drifting at $10 \%$, or "mixed" (see Figure 1A). The "mixed" standard consisted of a sequence of static and drifting subintervals. A static interval always followed a drifting one (and vice versa) and the total duration of static and drifting subintervals was always the same across trials (50\% static, 50\% drifting over the whole standard duration), but we assigned a duration between 100 and $200 \mathrm{~ms}$ to each subinterval, randomly, within, and across trials. A 500-ms blank interval separated the two tests and the relative spatial position of the stimuli was randomized. The presentation order of the two stimuli was also randomized and we saved the trials in which the standard was displayed first separately from those in which it was displayed second for later analysis. Subjects had to report which of the two tests seemed to have the longer duration. The data were fitted with cumulative Gaussian functions that were free to vary in position and width (see Figure 2C for example psychometric functions). The $50 \%$ point on the psychometric function (point of subjective equality, PSE) provided an estimate of the perceived duration of the standard stimulus. The discrimination threshold provided a measure of precision. The discrimination threshold was defined as the width of the error distribution $\sigma$. The value of $\sigma$ is equivalent to the difference between the 50 and $84 \%$ point on the psychometric function.

\section{RESULTS}

We asked our subjects to compare the relative duration of two sequentially displayed temporal intervals (containing sinusoidal gratings modulated in luminance, Figure 1B). In different conditions (Figure 1A), we modulated the number of temporal changes contained in the standard test (fixed duration across trials), which could be static, drifting, or mixed (composed of an alternation of static and drifting intervals of randomly varied duration, thus containing an intermediate number of changes between the static and the drifting stimulus). The other test (the comparison, variable duration across trials) was always drifting. Since we used three standard durations $(600,1200$, and $2400 \mathrm{~ms})$, we needed to transform the data in order to explore the effects of standard type and duration on apparent duration and discriminability. We divided the PSEs by the correspondent standard durations and then subtracted 1 . The values we obtained described the proportion of duration change relative to the standard duration: negative values indicated compression, positive values expansion, zero corresponds to the actual duration (Figure 2B). The discrimination thresholds were divided by the correspondent
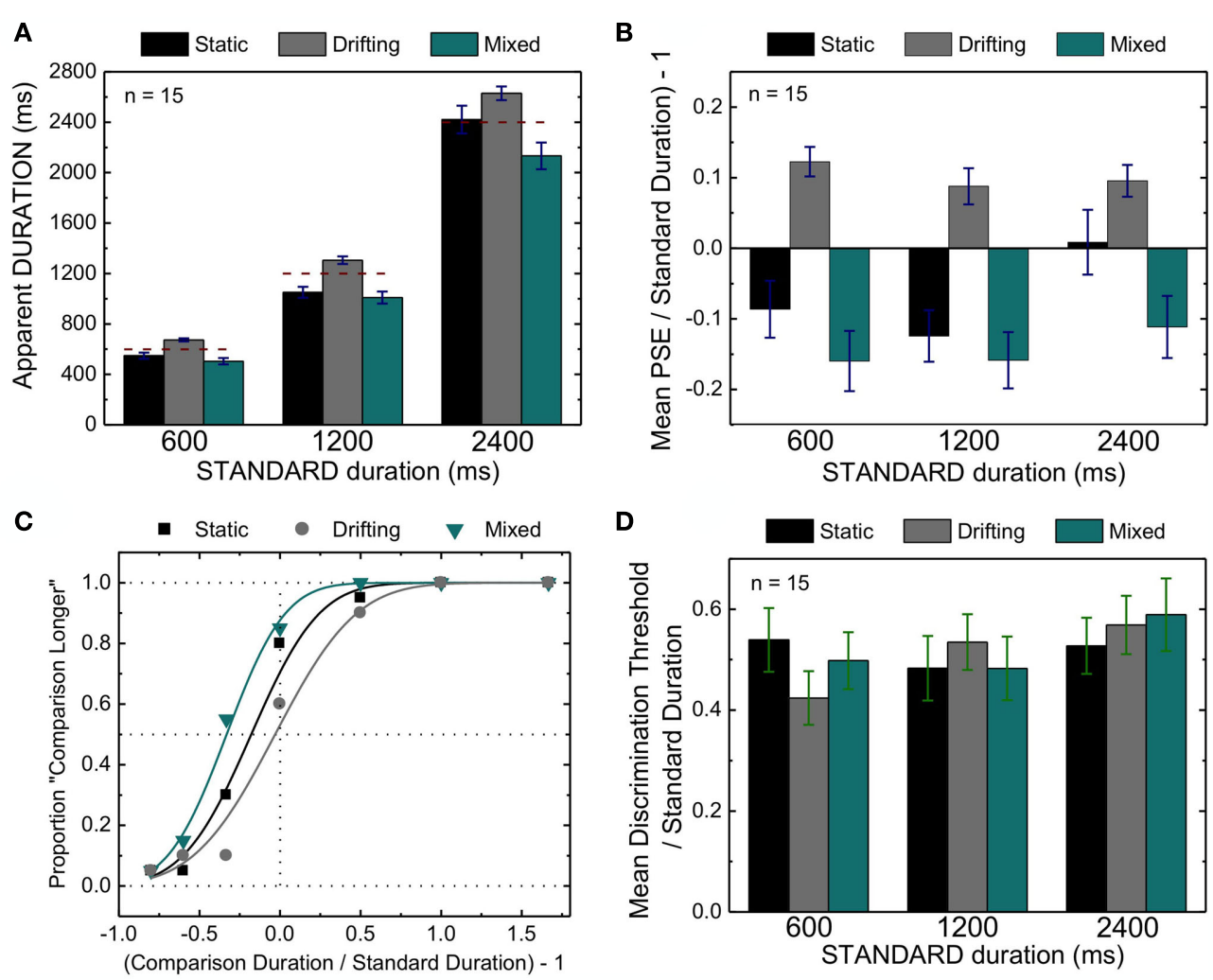

FIGURE 2 | Effect of stimulus change on apparent duration and discrimination thresholds. (A) Mean perceived duration (calculated as the $50 \%$ point on the psychometric function, PSE) across 15 subjects for three standard durations and three stimulus configurations. Dashed lines represent the actual standard durations. Error bars indicate \pm 1 SEM. (B) Proportion change in perceived duration relative to the true standard duration [calculated from the data plotted in (A)] for the same conditions as in (A). (C) Example psychometric curves for a naive observer for the three stimulus configurations and for $600 \mathrm{~ms}$ standard duration. (D) Average discrimination thresholds (defined as the difference between the 50 and $84 \%$ point on the psychometric function/the standard duration) for the same subjects and conditions as in (A). Error bars indicate \pm 1 SEM. 
standard durations to give Weber fractions. In Figure 2A we plotted the actual PSE and discrimination threshold values to facilitate an intuitive reading of the main results. Figure $2 \mathrm{C}$ shows typical psychometric functions for a naïve observer for the $600-\mathrm{ms}$ conditions.

Statistical analyses conducted on the ratios revealed significant main effects of both standard stimulus type [General Linear Model repeated measures, $F(2)=21.317, p<0.001]$ and standard duration $[F(2)=4.726, p=0.017]$ on perceived duration (PSEs, Figure 1A). In general, we observed time compression for the Static $(\sim-10 \%)$ and Mixed conditions $(\sim-15 \%)$ relative to the actual duration, while the Drifting standard appeared expanded $(\sim+10 \%)$. When the different stimulus types were pooled together, we found the estimates for the 2400-ms condition to be veridical, but there was a slight compression for both the $600-(\sim-4 \%)$ and $1200-\mathrm{ms}$ data $(\sim-6.5 \%)$. Moreover, the effect of standard duration depends on stimulus type (significant interaction, $p=0.039$ ). More specifically, we found that, for all the standard durations, the Mixed stimulus appeared significantly compressed relative to the Drifting stimulus (paired $t$-tests, all $p<0.001)$. No significant difference emerged from the comparison between the Static and Mixed conditions (paired $t$-tests, all $p>0.1$, Not Significant). Confirming previous reports (Brown, 1995; Kanai et al., 2006; Kaneko and Murakami, 2009), the Static standard looked shorter-lived than the Drifting comparison for all standard durations (all $p<0.001$ ) apart from $2400 \mathrm{~ms}$ ( $p=0.102$, Not Significant). One might argue that the duration estimates for the Mixed stimulus (which contains static and drifting intervals in equal proportions) could result from a linear combination of the estimates of its static and drifting components. However, the apparent duration of the Mixed stimulus was found to be significantly less than the average of the Static and Drifting judgments for all standard durations (paired $t$-tests, all $p<0.01)$. A mild, but significant expansion was observed for the drifting conditions relative to the veridical standard duration (onesample $t$-tests conducted on the ratios against 0 , all $p<0.01$ ). This may be explained by the fact that we had to increase the range for long comparison durations, which, in an initial pilot experiment, were not reliably estimated for most of the subjects, especially in the 2400-ms standard duration. Consequently, the mean of the comparison durations, which has been shown to influence subjects' judgments (Nachmias, 2006; Lapid et al., 2008), corresponded to a higher value than the true standard duration.

The discrimination thresholds are shown in Figure 2D. Analyses conducted on the Weber thresholds (discrimination threshold/standard duration), revealed no significant effect of the stimulus type on duration judgment accuracy. However, the average discrimination threshold for the 2400-ms standard duration (0.56) was higher than those for 600 and $1200 \mathrm{~ms}$ (both 0.5) resulting in a significant main effect for standard duration [General Linear Model repeated measures, $F(2)=4.278, p=0.024]$ and a significant interaction with stimulus type $[F(4)=2.704, p=0.039]$. No significant difference emerged from paired comparisons of static, drifting, and mixed conditions for the three standard durations separately.
For all the conditions, we interleaved trials in which the standard was displayed first with trials in which it was displayed second. Since it has been shown (Jamieson and Petrusic, 1975; Nachmias, 2006; Lapid et al., 2008) that the stimulus order has an influence on both apparent duration (PSE) and discrimination threshold, we reanalyzed the data with presentation order as a variable with two levels (standard first and standard second). Figure 3A contains a graphic representation of this separation for the transformed PSEs [PSE/(standard duration) - 1]. Consistent with previous reports (Jamieson and Petrusic, 1975; Nachmias, 2006; Lapid et al., 2008), it is evident that duration estimates were significantly lower when the standard was displayed first relative to when it was displayed second [General Linear Model, main effect for standard order, $F(1)=13.433, p<0.01]$. The novel finding is that the difference between the two-stimulus orders significantly increases with standard duration [interaction standard order $\times$ standard duration, $F(2)=23.058, p<0.001]$. Also, the standard order has a different influence on apparent duration depending on stimulus type [interaction standard order $\times$ standard type, $F(2)=7.157, p<0.01]$. In particular the difference between standard first and standard second seems less pronounced for the mixed condition relative to static or drifting.

In Figure 3B, we plotted the discrimination thresholds for different standard orders and durations. The difference between presentation orders is clear: accuracy is statistically higher (lower discrimination threshold) for standard first condition [General Linear Model, $F(1)=26.849, p<0.001]$. However, in this case, this difference does not depend on standard type or duration (all interactions not significant).

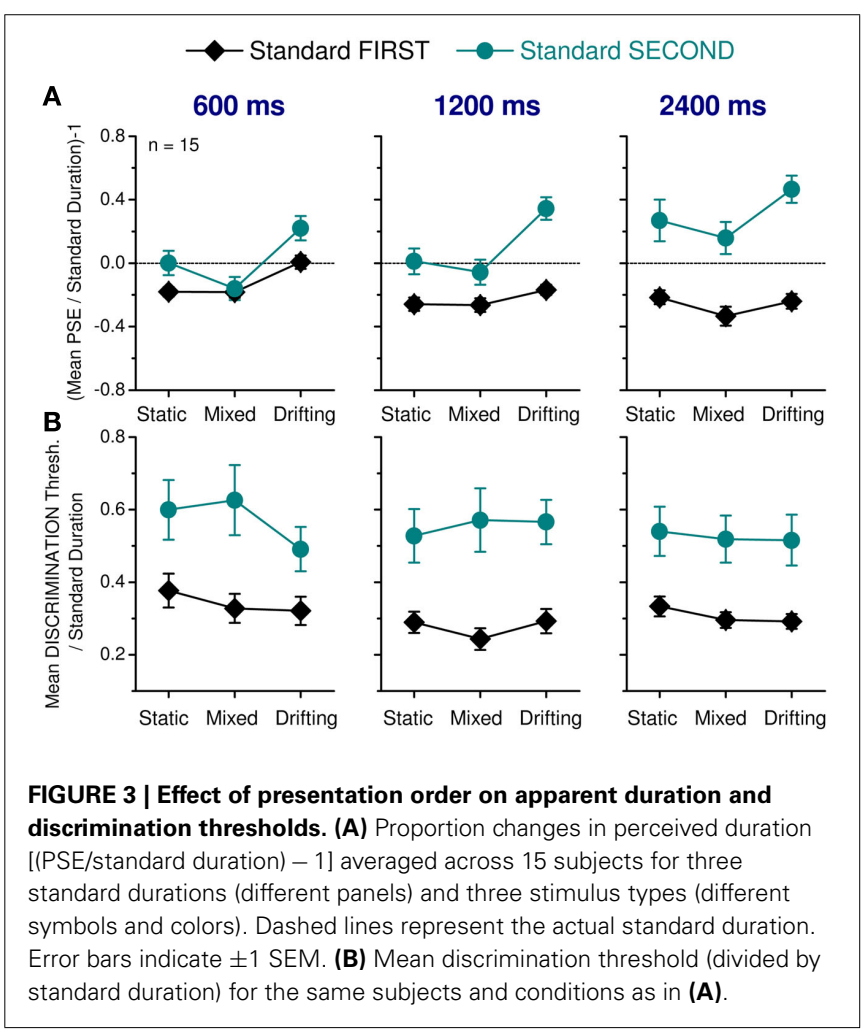




\section{DISCUSSION}

We investigated the effect of the number of temporal changes and order on the apparent duration of visual intervals that were at most a few seconds long. We found that:

- the duration of a stimulus composed of a random alternation of static and drifting intervals (mixed standard) appeared compressed either relative to a drifting comparison stimulus or to the average of the static and drifting estimates.

- a static stimulus appeared shorter in duration than a drifting stimulus, confirming previous observations (Brown, 1995; Kanai et al., 2006; Kaneko and Murakami, 2009).

- no substantial difference in duration discriminability emerged between the three presentation conditions. However, lower discriminability was associated with $2400 \mathrm{~ms}$ relative to 600 and $1200 \mathrm{~ms}$ standard durations.

- the duration of the first of a pair of sequentially displayed stimuli appears compressed and the magnitude of the effect increases with standard stimulus duration.

- duration discrimination was significantly better for the first presented stimulus relative to the second regardless of standard duration.

The dilation effect induced by speed or temporal frequency on the perceived duration of a visual stimulus has been traditionally seen as a supporting evidence for a change-based theory of time perception (Fraisse, 1963; Poynter, 1989). This model posits that our experience of time strongly relies on the amount of temporal information contained in the interval we want to estimate. More specifically, a prominent role is assigned to the extent to which an interval can be segmented into a sequence of temporal changes or features (Poynter, 1983; Poynter and Homa, 1983). The changes in spatial position that occur within the trajectory of a visual stimulus in motion have been considered to provide a good basis on which to test of the predictions of this model. For instance, Brown (1995) investigated duration estimates for stimuli that translated across a monitor along a non-linear trajectory at different speeds. He observed an apparent duration expansion for moving relative to stationary stimuli that increased with stimulus speed. According to the author, this result supports the change model, as faster stimuli change spatial location more frequently than slower ones within the same time interval. In our experiment, the number of changes associated with the mixed stimulus should arguably be intermediate between the stationary stimulus and the continuously drifting one. Therefore, according to the change model, we should expect the duration estimates for the mixed stimulus to be somewhere halfway between those for the stationary and the drifting stimuli. However, this is not the case, as the mixed stimulus appears to be more short-lived than the mean of the other two conditions and is not perceived as significantly longer than the stationary stimulus. One might argue that our stimuli (drifting gratings) do not change in spatial location and therefore they might not be readily segmented into subunits. However, the findings that a similar increase in perceived duration to that observed for translating stimuli also occurs for flickering stimuli (Goldstone and Lhamon, 1974; Lhamon and Goldstone, 1974; Kanai et al., 2006) and for drifting Gabors (Kaneko and Murakami, 2009), which do not contain changes in spatial location, argue against this view. Kaneko and Murakami (2009) proposed a slightly different account for the speed-induced time expansion. According to their view, the rate at which our internal clock (Creelman, 1962; Treisman, 1963; Treisman et al., 1990) produces ticks is increased with stimulus speed in order to increase the temporal resolution of the system. As far as our stimuli are concerned, the predictions of this model do not substantially differ from those of the classic change model, as the increase in the tick rate associated with the intervals containing drifting motion should be halved for the mixed stimulus (which contains 50\% drifting and 50\% static intervals). Interestingly, stimuli moving at a constant speed are not perceived as having the same duration as accelerating or decelerating stimuli with the same average speed (Matthews, 2011), again arguing against the idea of duration as a reflection of an accumulation of temporal information.

Kanai et al. (2006) reported that the dependency of perceived duration of expanding gratings on temporal frequency tends to saturate quite quickly, in a range between 4 and $8 \mathrm{~Hz}$. The temporal frequency of our drifting stimuli $(10 \mathrm{~Hz})$ falls slightly outside this range, therefore it is arguable that different mechanisms might be at work for low relative to high temporal frequencies and we are maybe tapping into a range of frequencies that is not substantially influenced by the change rate within an interval. However, Kaneko and Murakami (2009), using stimuli that were more similar to ours (drifting Gabors), reported that duration overestimation increased linearly with $\log$ speed even for values higher than $10^{\circ} \%$ s. Since the spatial frequency of our stimuli was always $1 \mathrm{cycle} /{ }^{\circ}$ we cannot discriminate between the effect of temporal frequency and speed on apparent duration.

The inter-stimulus interval (ISI) between standard and comparison stimuli was kept constant at $500 \mathrm{~ms}$ across trials and we did not use a mask to prevent visual aftereffects. However, the spatial position and the presentation order of the stimuli were fully randomized across trials. The standard and the comparison could be either displayed to the right or to the left of the fixation dot. Therefore, if there is an influence of visual aftereffects on perceived duration in our setup, it can be argued that it should equally affect both the standard and the comparison stimuli. We decided to use only two (right and left) rather than many possible spatial positions to keep the task as simple as possible and avoid attention-related effects. Judging duration is an attentionally demanding task that naïve subjects in particular find quite challenging. First, observers have to allocate their attention to the considered stimulus interval in a given spatial position and then they have to sustain their attention until the very end of the interval, unlike in speed, or orientation judgments tasks, for example, in which attention is only required until a decision is made. Therefore, the use of only two predictable spatial positions, allowed us to minimize the attentional resources required to relocate our spatial attention to unpredictable spatial positions.

We instructed subjects to fixate the dot located at the center of screen for the whole duration of the experiment. However, one might argue that some of our conditions could have induced reflexive saccades, although reflexive saccades were not spontaneously reported by observers. A visual interval displayed at the time of a saccadic eye-movement appears compressed relative to 
an interval displayed well before the saccadic onset (Morrone et al., 2005). In our experiment, the condition which might attract most saccades is the continuously drifting one, which, for the aforementioned reasons, should therefore be perceived as compressed relative to, for example, the stationary condition. However, the opposite was true, as the drifting stimulus was actually perceived as longer than the static (or the mixed) one. Also, the mixed stimulus does not appear more compressed than the static one, even though it might attract more reflexive saccades. Therefore, the presence of reflexive saccades is likely to work against the pattern of results we observed.

The stimuli we used in this study do not only differ in terms of the number of changes they contained, but also in terms of the predictability of subsequent frames of a motion sequence. A recent model proposed by Johnston (2010) posits that the system might use a "predict-and-compare" strategy to determine the duration of a brief interval. In a predict-and-compare clock the clock produces a prediction of the visual world's appearance after a given time (for example, $100 \mathrm{~ms}$ ) and then continuously compares it with the current appearance of the world that comes from the sensory input. Once the current appearance of the world matches the prediction, the system knows that $100 \mathrm{~ms}$ have passed, it sends a tick to the accumulator and then it resets the prediction. Clearly, the internal predictability of an interval in time plays an important role in the ability of the model to determine its duration. In the present study, we used some stimuli that could not benefit from a predict-and-compare strategy (static), stimuli with a high degree of predictability throughout the interval (drifting at $10 \%$ ) or a low degree of predictability at transitions (mixed). The poorly predictable stimulus was a balanced mixture of static and drifting subintervals; each of them had a randomly chosen duration and a static interval always followed a drifting one. In other words, the model could not predict the appearance of the stimulus at the end of a segment solely on the basis of its temporal frequency or speed, therefore we expected it might provide an inaccurate estimate of duration. Static stimuli would have to be processed by a generic mechanism. We observed that the poorly predictable stimulus (mixed condition, Figure 2A) was perceived to be compressed relative to the highly predictable drifting stimulus. The difference in apparent duration between static and drifting conditions suggest the use of different mechanism in the two cases. Critically, the duration of the mixed stimulus appeared reduced relative to the average of the drifting and static components, implying that the system does not simply integrate these intervals linearly to determine duration. The unpredictable change at the stimulus transition appears to introduce a small reduction in perceived duration that cannot simply be attributed to combining the static and moving intervals.

Another recent model by Ahrens and Sahani (2011) uses the content of an interval to estimate time. This model assumes that the duration of an interval is derived from a Bayesian combination of a sensory-based estimate of dynamic stimulus statistics with an internal estimate that is independent of stimulus properties. More specifically, changes in the stimulus detected by different stochastic sensory streams (which correspond to retinal snapshots of the stimulus taken at different points in time) are integrated to provide an estimate of duration that is based on the similarity between successive snapshots (the bigger the change between them, the longer the time period that is estimated to separate them). Therefore, the model predicts an underestimation of the duration of a static stimulus relative to a drifting one (as is observed in our results), but it is not clear how it can account for the lack of a substantial difference between the static and the mixed stimulus. Also, the model predicts a lower precision for the static (the duration of which is determined solely on the basis of the internal estimate) relative to the drifting stimuli (which can also take advantage of the stimulus-driven estimate). This prediction was confirmed experimentally, however, we did not observe a difference in the discrimination threshold between the two-stimulus types in our study (Figure 2D).

Fechner was the first to notice that when a subject was asked to lift two weights sequentially, the magnitude of the first one was underestimated (Fechner, 1860; Woodrow, 1951; Woodworth and Schlosberg, 1954). This classic order effect was also replicated in the time domain by the demonstration that the first of a pair of brief visual stimuli was perceived as shorter in duration than the second one (Jamieson and Petrusic, 1975). Interestingly, the ability to discriminate between two stimuli is also affected by the presentation order. More specifically, discrimination thresholds are lower for the first of a pair of sequentially displayed tests, regardless of whether the presentation order is blocked or randomized (Nachmias, 2006). Lapid et al. (2008) showed that this effect also holds for duration. Using both auditory and visual stimuli, they found that the just noticeable difference (JND) is smaller (i.e., higher discriminability) when a stimulus is displayed first. The general explanation for these order effects on PSEs and JNDs is that subjects tend to refer to an internal implicit standard for their judgments rather than to the presented standard, as suggested by the similarities observed in discriminability and perceived magnitude between single-stimulus tasks (in which no standard was presented and, therefore, subjects had to refer to an internal standard) and two-stimulus tasks when the standard was presented first. In all the conditions of the present experiments, we randomized the presentation order and collapsed together trials in which the standard was displayed first with trials where it was displayed second to minimize these biases. However, since we used quite a wide range of durations, we were also interested to investigate whether the time order effects vary with the presentation time. Jamieson and Petrusic (1975) used pairs of intervals in the range $240-5515 \mathrm{~ms}$, but they simply determined the proportion of correct responses rather than deriving a psychometric function. Our finding that the standardized difference in PSE (PSE/standard duration) between standard first and standard second conditions significantly increased with standard duration (Figure 3A) might suggest that that the remembered value of the first duration decays over time although, the observation that the time order effect declines as the ISI increases (Jamieson and Petrusic, 1975) seems to argue against this conclusion. It may be that both retaining a duration estimate and extracting a new duration estimate are attention demanding and that competition for limited resources generates a reduction in the capacity to sustain the perceived magnitude of the stored interval without any increase in the uncertainty about its magnitude. We also observed a smaller difference in PSE between standard first and standard second for the mixed stimulus, 
suggesting that with a less predictable stimulus, subjects seemed to be less prone to ignore the presented standard and refer to an internalized reference to make a duration judgment.

In conclusion, we showed that the number of temporal features that are contained within an interval are not necessarily the main factor determining the apparent duration of dynamic stimuli. Therefore, speed-related changes in perceived time cannot be

\section{REFERENCES}

Ahrens, M. B., and Sahani, M. (2011). Observers exploit stochastic models of sensory change to help judge the passage of time. Curr. Biol. 21, 200-206.

Ayhan, I., Bruno, A., Nishida, S., and Johnston, A. (2011). Effect of the luminance signal on adaptationbased time compression. J. Vis. 11, 22.

Ayhan, I., Bruno, A., Nishida, S. Y., and Johnston, A. (2009). The spatial tuning of adaptation-based time compression. J. Vis. 9, 1-12.

Block, R. A., and Reed, M. A. (1978). Remembered duration: evidence for a contextual-change hypothesis. $J$. Exp. Psychol. Hum. Learn. Mem. 4, 656-665.

Brown, J. F. (1931). On time perception in visual movement fields. Psychol. Res. 14, 233-248.

Brown, S. W. (1995). Time, change, and motion: the effects of stimulus movement on temporal perception. Percept. Psychophys. 57, 105-116.

Bruno, A., Ayhan, I., and Johnston, A. (2010). Retinotopic adaptationbased visual duration compression. J. Vis. 10, 30

Bruno, A., Ayhan, I., and Johnston, A. (2011). Duration expansion at low luminance levels. J. Vis. 11(14):13, $1-13$.

Bruno, A., and Johnston, A. (2010). Contrast gain shapes visual time. Front. Psychol. 1:170. doi:10.3389/fpsyg.2010.00170

Burr, D., Tozzi, A., and Morrone, M. C. (2007). Neural mechanisms for timing visual events are spatially selective in real-world coordinates. Nat. Neurosci. 10, 423-425.

Cicchini, G. M., and Morrone, M. C. (2009). Shifts in spatial attention affect the perceived duration of events. J. Vis. 9, 1-13.

Creelman, C. D. (1962). Human discrimination of auditory duration. $J$. Acoust. Soc. Am. 34, 582-593.
Eagleman, D. M., and Pariyadath, V. (2009). Is subjective duration a signature of coding efficiency? Philos. Trans. R. Soc. Lond. B Biol. Sci. 364, 1841-1851.

Fechner, G. T. (1860). Elemente der Psychophysik. Leipzig: Breitkopf \& Hartel.

Fraisse, P. (1963). The Psychology of Time. New York: Harper and Row.

Goldstone, S., and Lhamon, W. T. (1974). Studies of auditory-visual differences in human time judgment. 1. Sounds are judged longer than lights. Percept. Mot. Skills 39, 63-82.

Jamieson, D. G., and Petrusic, W. M. (1975). Presentation order effects in duration discrimination. Percept. Psychophys. 17, 197-202.

Johnston, A. (2010). "Modulation of time perception by visual adaptation," in Attention and Time, eds A. C. Nobre and J. T. Coull (Oxford: Oxford University Press), 187-200.

Johnston, A., Arnold, D. H., and Nishida, S. (2006). Spatially localized distortions of event time. Curr. Biol. 16, 472-479.

Johnston, A., Bruno, A., Watanabe, J., Quansah, B., Patel, N., Dakin, S., and Nishida, S. (2008). Visually-based temporal distortion in dyslexia. Vision Res. 48, 1852-1858.

Kanai, R., Paffen, C. L., Hogendoorn, H., and Verstraten, F. A. (2006). Time dilation in dynamic visual display. $J$. Vis. 6, 1421-1430.

Kaneko, S., and Murakami, I. (2009). Perceived duration of visual motion increases with speed. J. Vis. 9, 14.

Lapid, E., Ulrich, R., and Rammsayer, T. (2008). On estimating the difference limen in duration discrimination tasks: a comparison of the 2AFC and the reminder task. Percept. Psychophys. 70, 291-305.

Leiser, D., Stern, E., and Meyer, J. (1991). Mean velocity and total time

explained in this way and we require a different means of incorporating content-dependence into a time estimation model, such as the ability to predict the appearance of a stimulus forward in time.

\section{ACKNOWLEDGMENTS}

We would like to thank Yi Wu for her valuable help in the data collection. This study was supported by The Wellcome Trust.

estimation effects of order and proportions. J. Environ. Psychol. 11, 347-358.

Lhamon, W. T., and Goldstone, S. (1974). Studies of auditory-visual differences in human time judgment. 2. More transmitted information with sounds than lights. Percept. Mot. Skills 39, 295-307.

Matthews, W. J. (2011). How do changes in speed affect the perception of duration? J. Exp. Psychol. Hum. Percept. Perform. 37, 1617-1627.

Morrone, M. C., Ross, J., and Burr D. (2005). Saccadic eye movements cause compression of time as well as space. Nat. Neurosci. 8, 950-954.

Nachmias, J. (2006). The role of virtual standards in visual discrimination. Vision Res. 46, 2456-2464.

Pariyadath, V., and Eagleman, D. (2007) The effect of predictability on subjective duration. PLoS ONE 2, e1264. doi:10.1371/journal.pone.0001264

Pariyadath, V., and Eagleman, D. M (2008). Brief subjective durations contract with repetition. J. Vis. 8 , $11-16$.

Poynter, W. D. (1983). Duration judgment and the segmentation of experience. Mem. Cognit. 11, 77-82.

Poynter, W. D. (1989). "Judging the duration of time intervals: a process of remembering segments of experience," in Time and human cognition: A life-span perspective, Vol. 59, eds I. Levin and D. Zakay (Amsterdam: Elsevier), 305-321.

Poynter, W. D., and Homa, D. (1983). Duration judgment and the experience of change. Percept. Psychophys. $33,548-560$.

Roelofs, C. O., and Zeeman, W. P. C. (1951). Influence of different sequences of optical stimuli on the estimation of duration of a given interval of time. Acta Psychol. (Amst.) 8, 89-128.

Treisman, M. (1963). Temporal discrimination and the indifference interval. Implications for a model of the "internal clock." Psychol. Monogr. 77, 1-31.

Treisman, M., Faulkner, A., Naish, P. L., and Brogan, D. (1990). The internal clock: evidence for a temporal oscillator underlying time perception with some estimates of its characteristic frequency. Perception 19, 705-743.

Tse, P. U., Intriligator, J., Rivest, J., and Cavanagh, P. (2004). Attention and the subjective expansion of time. Percept. Psychophys. 66, 1171-1189.

Woodrow, H. (1951). "Time perception," in Handbook of Experimental Psychology, eds S. S. Stevens and H. S. Langfeld (New York: John Wiley), 1224-1236.

Woodworth, R. S., and Schlosberg, H. (1954). Experimental Psychology. New York: Holt.

Conflict of Interest Statement: The authors declare that the research was conducted in the absence of any commercial or financial relationships that could be construed as a potential conflict of interest.

Received: 03 January 2012; paper pending published: 01 February 2012; accepted: 08 March 2012; published online: 21 March 2012.

Citation: Bruno A, Ayhan I and Johnston A (2012) Effects of temporal features and order on the apparent duration of a visual stimulus. Front. Psychology 3:90. doi: 10.3389/fpsyg.2012.00090

This article was submitted to Frontiers in Perception Science, a specialty of Frontiers in Psychology.

Copyright () 2012 Bruno, Ayhan and Johnston. This is an open-access article distributed under the terms of the Creative Commons Attribution Non Commercial License, which permits noncommercial use, distribution, and reproduction in other forums, provided the original authors and source are credited. 